\title{
CIRCLE ACTIONS ON HOMOTOPY SPHERES BOUNDING PLUMBING MANIFOLDS
}

\author{
REINHARD SCHULTZ ${ }^{1}$
}

\begin{abstract}
Smooth circle actions are constructed on certain homotopy spheres not previously known to admit such actions.
\end{abstract}

In this paper we shall prove the following two results:

Proposition A. Let $\Sigma^{8}$ be any homotopy 8-sphere. Then there is a smooth semifree circle action on $\Sigma^{8}$ with $S^{4}$ as its fixed point set.

Proposition B. Let $\sum^{10}$ be any homotopy 10-sphere bounding a spin manifold. Then there is a smooth semifree circle action on $\Sigma^{10}$ with $S^{4}$ as its fixed point set.

Combining these with other results, we know that any smooth manifold $\Sigma^{n}$ which is piecewise-differentiably homeomorphic to $S^{n}$, bounds a spin manifold, and satisfies $n \leqq 13$ has a smooth circle action. The above propositions imply the cases $n=8,10$, while the cases $n=7,11,12$ follow because $\Gamma_{n}=\partial P_{n+1}$ in these cases and every homotopy sphere in $\partial P_{n+1}(n \geqq 5)$ has a semifree circle action with a homotopy $(n-4)$-sphere as its fixed point set (e.g., see [3]). Finally, the cases $n=9,13$ follow from the above remark on $\partial P_{n+1}$ and results of Bredon [1].

Undoubtedly, the central difficulty in obtaining connected Lie group actions on homotopy spheres is the lack of a manageable construction for an arbitrary such manifold. The value of such a realization is obvious in the construction of large orthogonal group actions on homotopy spheres bounding $\pi$-manifolds. Bredon's construction of smooth $S^{1}$ and $S^{3}$ actions on homotopy spheres in the image of the Milnor-MunkresNovikov pairing [1] is another illustration of the usefulness of an explicit construction for a given homotopy sphere. In this paper we shall show that certain homotopy spheres in the image of the Milnor plumbing pairing $\sigma_{p, q}: \pi_{q}\left(S O_{p}\right) \times \pi_{p}\left(S O_{q}\right) \rightarrow \Gamma_{p+q+1}($ see [4] or [5]) also have smooth circle

Received by the editors July 26, 1971 and, in revised form, March 24, 1972.

AMS 1970 subject classifications. Primary 57D60, 57E15, 57E25; Secondary 57D50, $57 \mathrm{D} 55$.

Key words and phrases. Homotopy sphere, smooth semifree circle action, plumbing pairing, equivariant diffeomorphism, Puppe sequence, $k$-invariant, localized space.

1 The author was partially supported by NSF grant GP-19530. 
actions. Propositions A and B follow from this, results of D. Frank [4] stating that the image of $\sigma_{3,4}$ is $\Gamma_{8}$ and the image of $\sigma_{3,6}$ is $2 \Gamma_{10}$, and some homotopy-theoretic computations. I am indebted to D. Frank for suggesting that results like Propositions $\mathrm{A}$ and $\mathrm{B}$ might be obtainable from [4].

1. Constructing semifree circle actions. We shall construct smooth semifree circle actions on certain homotopy spheres in the image of $\sigma_{p, q}$ by the familiar technique of pasting two $S^{1}$ manifolds together via an equivariant diffeomorphism. However, we shall be pasting along a semifree $S^{1}$ manifold rather than a free $S^{1}$ manifold (which is the usual way of studying semifree actions; e.g., see [2, Chapter III]).

Let $p, q$ and $r$ be positive integers satisfying $2 r \leqq q+1$. Then $S^{1}$ acts orthogonally on $R^{q^{+1}}$ via the representation containing $r$ copies of the standard 2-dimensional representation and a trivial representation; call this representation $A_{r}$. Then the induced smooth action on $S^{p} \times S^{q}$ given by

$$
z \cdot(x, y)=\left(x, A_{r}(z) y\right), \quad x \in S^{p}, y \in S^{q}
$$

is semifree, and its fixed point set is $S^{p} \times S^{q-2 r}$.

The orthogonal action $A_{r}$ on $S^{q}$ may be readily interpreted in terms of the homeomorphism from $S^{q}$ to the join $S^{2 r-1} * S^{q-2 r}$; namely, it is the free linear action in the first join coordinate (an element of $S^{2 r-1}$ ) and the trivial action on the other two join coordinates (elements of $S^{q-2 s}$ and $[0,1]$ respectively). One advantage of this interpretation is that the orbit space projection is immediately recognized as the join of the canonical map $p_{r-1}: S^{2 r-1} \rightarrow C P^{r-1}$ with the identity on $S^{q-2 r}$. It is well known that this map is homotopically equivalent to the $(q-2 r+1)$-fold suspension of $p_{r-1}$.

Lemma 1.2. Let $\alpha \in \pi_{p}\left(S O_{q}\right)$ be in the image of $\pi_{p}\left(U_{r}\right)$ and let $\beta \in$ $\pi_{q}\left(S O_{p}\right)$ be in the image of

$$
\left(S^{q-2 r+1} p_{r-1}\right)^{*}:\left[S^{q-2 r+1} C P^{r-1}, S O_{p}\right] \rightarrow \pi_{q}\left(S O_{p}\right) .
$$

Then the homotopy $(p+q+1)$-sphere corresponding to $\sigma_{p, q}(\alpha, \beta)$ has a semifree circle action whose fixed point set is an ordinary $(p+q+1-2 r)$ sphere.

PROOF. Let $\varphi: S^{p} \rightarrow U_{r}$ be a smooth map representing $\alpha$ and let $f$ be the diffeomorphism of $S^{p} \times S^{q}$ defined by $f(x, y)=(x, \varphi(x) y)$, where $U_{r}$ acts orthogonally on $S^{q}$; then $f$ is equivariant with respect to the $S^{1}$ action defined in (1.1). Let $\psi: S^{a} \rightarrow S O_{p}$ be a continuous map representing $\beta$; the assumption that $\beta$ factors through $S^{q-2 r+1} C P^{r-1}$ implies that $\psi$ may be assumed to be constant on the orbits of the action $A_{r}$. If $S O_{p}$ is taken with 
the trivial $S^{1}$ action, then the smooth equivariant approximation theorem $[7,1.12]$ implies that $\psi$ may also be assumed to be smooth. Hence the diffeomorphism $g(x, y)=(\psi(y) x, y)$ is also equivariant with respect to the $S^{1}$ action of (1.1); therefore, the commutator $[f, g]$ is also $S^{1}$ equivariant.

The action given in (1.1) obviously extends to smooth $S^{1}$ actions on the manifolds $D^{p+1} \times S^{q}$ and $S^{p} \times D^{q+1}$. Thus if these two manifolds with boundary are pasted together via the equivariant diffeomorphism $[f, g]$, the resulting closed manifold has a smooth circle action. But the resulting closed manifold is merely the homotopy sphere corresponding to $\sigma(\alpha, \beta)$; e.g., see [5, remark preceding 2.1]. Since the fixed point sets of the extended actions on $D^{p+1} \times S^{q}$ and $S^{p} \times D^{q+1}$ are $D^{p+1} \times S^{q-2 r}$ and $S^{p} \times S^{q-2 r}$ respectively, the fact that $[f, g]$ is the identity on $S^{p} \times S^{q-2 r}$ implies that the fixed point set of the action on $\sigma(\alpha, \beta)$ is the ordinary sphere

$$
S^{p+q+2 r+1}=D^{p+1} \times S^{q-2 r} \cup S^{p} \times D^{q-2 r+1} .
$$

It follows immediately that the circle action constructed above is semifree.

2. Proof of main results. The proofs of Propositions A and B are roughly parallel, although the latter is somewhat more complicated.

Proof of Proposition A. Let $\alpha \in \pi_{3}\left(\mathrm{SO}_{4}\right)$ map to the generator of $\pi_{3}(S O)=Z$, let $\beta$ generate $\pi_{3}\left(S O_{3}\right)=Z$, and let $\eta$ generate $\pi_{4}\left(S^{3}\right)=Z_{2}$. According to [4, Example 1, p. 565], the element $\sigma_{3,4}(\alpha, \beta \eta) \in \Gamma_{8}$ is nonzero for any choice of $\alpha$. On the other hand, it is well known that $\alpha$ may be chosen to lie in the image of $\pi_{3}\left(U_{2}\right)$. Since $\eta$ is the orbit space projection $S^{0} * S^{3} \rightarrow S^{0} * S^{2}$ described in $\S 1$, Proposition A follows immediately from Lemma 1.2 .

The proof of Proposition B depends on homotopy computations involving 3-primary components; it will be convenient to localize all spaces at the prime 3 . A discussion of localization functors appears in [6, Chapter II]; the main property we need is that the topological localization map $l_{X}: X \rightarrow X_{(p)}$ is given in homotopy by the algebraic localization $\pi_{*}(X) \rightarrow \pi_{*}(X) \otimes Z_{(p)}$. The following generalization is well known.

Lemma 2.1. Let $X$ be a finite $C W$ complex and let $Y$ be a topological group, so that $[S X, Y]$ is naturally an abelian group. Then there is a natural isomorphism $\alpha_{X}:\left[S X, Y_{(p)}\right] \rightarrow[S X, Y] \otimes Z_{(p)}$ such that $\alpha_{X} l_{X}$ * is the canonical map $[S X, Y] \rightarrow[S X, Y] \otimes Z_{(p)}$.

We shall need the following computational result:

LEMMA 2.2. Let $v^{\prime}$ generate $\pi_{6}\left(S_{(3)}^{3}\right)=Z_{3}$, and let $g: S^{5} \rightarrow C P^{2}$ be the canonical projection. Then $v^{\prime}$ is in the image of the map

$$
(S g)^{*}:\left[S C P^{2}, S_{(3)}^{3}\right] \rightarrow \pi_{6}\left(S_{(3)}^{3}\right) \text {. }
$$


Proof. Recall that the first two nonzero homotopy groups of $S_{(3)}^{3}$ are $Z_{(3)}$ in the dimension 3 and $Z_{3}$ in dimension 6 . These are related by the nontrivial $k$-invariant $P^{1} \in H^{7}\left(Z_{(3)}, 3 ; Z_{3}\right)=Z_{3}$.

By the exactness of the Puppe sequence and the mapping cone sequence

$$
S^{5} \stackrel{g}{\longrightarrow} C P^{2} \longrightarrow C P^{3} \stackrel{h}{\longrightarrow} S^{6},
$$

it suffices to show that $h^{*}: \pi_{6}\left(S_{(3)}^{3}\right) \rightarrow\left[C P^{3}, S_{(3)}^{3}\right]$ is the trivial map. But this is a straightforward consequence of the following two facts:

(i) $P^{1}$ is the first $k$-invariant of $S_{(3)}^{3}$.

(ii) The suspension of $P^{1}$ (i.e., $\iota^{3} \in H^{6}\left(Z_{(3)}, 2\right)$ ) has a nontrivial image in $H^{6}\left(C P^{3} ; Z_{3}\right)$.

REMARK. Since $S^{3}$ is the universal covering group of $S \mathrm{~S}_{3}$ and $\pi_{1}\left(\mathrm{SO}_{3}\right)=$ $Z_{2}$, there is an obvious homotopy equivalence from $S_{(3)}^{3}$ to $S O_{3(3)}$; consequently, the lemma remains true if $\mathrm{S}^{3}$ is replaced by $\mathrm{SO}_{3}$.

Proof of Proposition B. Let $\alpha \in \pi_{3}\left(\mathrm{SO}_{6}\right)=Z$ be a generator, and let $\beta$ generate the 3-primary component of $\pi_{6}\left(\mathrm{SO}_{3}\right)=Z_{12}$. As in the proof of Proposition $\mathrm{A}$, we know that $\alpha$ is in the image of $\pi_{3}\left(U_{3}\right)$. On the other hand, Lemma 2.2 implies that $\beta$ is in the image of $(\mathrm{Sg})^{*}:\left[\mathrm{SCP}^{2}, \mathrm{SO}_{3}\right] \rightarrow$ $\pi_{6}\left(\mathrm{SO}_{3}\right)$. Since $\sigma_{3,6}(\alpha, \beta)$ is nonzero by results of Frank [4, Example 2, p. 565], some exotic 10-sphere bounding a Spin manifold has a circle action of the desired type by Lemma 1.2. Since $b \operatorname{Spin}_{11}=Z_{3}$ is cyclic, an action on the other one may be constructed by taking an equivariant connected sum of this action with itself along the fixed point set.

\section{REFERENCES}

1. G. E. Bredon, $A \pi_{*}$-module structure for $\Theta_{*}$ and applications to transformation groups, Ann. of Math. (2) 86 (1967), 434-448. MR 36 \#4570.

2. W. Browder, Surgery and the theory of differentiable transformation groups, Proc. Conf. on Transformation Groups (New Orleans, La., 1967), Springer, New York, 1968, pp. 1-46. MR 41 \#6242.

3. W. Browder and T. Petrie, Semi-free and quasi-free $S^{1}$ actions on homotopy spheres, Essays on Topology and Related Topics (Mém. dédiés à Georges deRham), Springer, New York, 1970, pp. 136-146. MR 41 \#7716.

4. D. L. Frank, An invariant for almost-closed manifolds, Bull. Amer. Math. Soc. 74 (1968), 562-567. MR 36 \#5956.

5. R. Schultz, Composition constructions on diffeomorphisms of $S^{p} \times S^{q}$, Pacific J. Math. (to appear).

6. D. Sullivan, Geometric topology. I: Localization, periodicity, and Galois symmetry, M.I.T. Cambridge, Mass., 1970 (mimeographed).

7. A. G. Wasserman, Equivariant differential topology, Topology 8 (1969), 127-150. MR 40 \#3563.

Department of Mathematics, Purdue University, West Lafayette, Indiana 47907 